ARTICLE

Received 19 Sep 2013 | Accepted 27 Nov 2013 | Published 6 Jan $2014 \quad$ DOl: 10.1038/ncomms4027

\title{
Light-regulated gene repositioning in Arabidopsis
}

\author{
Chun-Miao Feng ${ }^{1}$, Yongjian Qiu¹, Elise K. Van Buskirk¹, Emily J. Yang ${ }^{1} \&$ Meng Chen ${ }^{1}$
}

Plant genomes are extremely sensitive to, and can be developmentally reprogrammed by environmental light cues. Here using rolling-circle amplification of gene-specific circularizable oligonucleotides coupled with fluorescence in situ hybridization, we demonstrate that light triggers a rapid repositioning of the Arabidopsis light-inducible chlorophyll $a / b$-binding proteins $(C A B)$ locus from the nuclear interior to the nuclear periphery during its transcriptional activation. $C A B$ repositioning is mediated by the red/far-red photoreceptors phytochromes (PHYs) and is inhibited by repressors of PHY signalling, including COP1, DET1 and PIFs. CAB repositioning appears to be a separate regulatory step occurring before its full transcriptional activation. Moreover, the light-inducible loci RBCS, PC and GUN5 undergo similar repositioning behaviour during their transcriptional activation. Our study supports a light-dependent gene regulatory mechanism in which PHYs activate light-inducible loci by relocating them to the nuclear periphery; it also provides evidence for the biological importance of gene positioning in the plant kingdom.

\footnotetext{
${ }^{1}$ Department of Biology, Duke University, Durham, North Carolina 27708, USA. Correspondence and requests for materials should be addressed to M.C. (email: meng.chen@duke.edu).
} 
C hanges in light quality and quantity trigger rapid alterations in gene expression and consequently lead to dramatic modifications in plant morphology and physiology ${ }^{1}$. For example, during seedling development, initial exposure to light turns on the photoautotrophic developmental programme called photomorphogenesis via massive transcriptional reprogramming ${ }^{2-4}$. A major class of light-induced nuclear genes encodes essential components of the chloroplast photosynthetic machinery, such as the chlorophyll $a / b$-binding proteins $(\mathrm{CABs})^{4}$. Although a suite of photoreceptors and their signalling components have been identified for mediating light responses, the mechanism by which light orchestrates complex developmental programmes such as photomorphogenesis is still poorly understood ${ }^{3}$.

Accumulating evidence from studies in yeast and metazoan models suggests that the organization of the genome, and in particular the spatial positioning of individual genes to distinct subnuclear compartments, plays an important role in cellular programming ${ }^{5-7}$. As the divergence of the plant kingdom predates the divergence of the fungal and metazoan lineages 8 , the general principles of gene positioning in yeast and metazoan models might not be conserved in plants. Therefore, we asked whether light regulates developmental programming by reorganizing the spatial positioning of individual light-responsive genes.

Compared with yeast and metazoan models, little is known about the positioning of individual genes in plants. A key obstacle for gene positioning studies in plants has been difficulty in labelling a single endogenous gene locus ${ }^{9}$. Although fluorescence in situ hybridization (FISH) has been successfully used in plants to study the organization of repetitive DNA and chromatin regions spanning more than $100 \mathrm{~kb}$ (refs 10-13), conventional FISH does not efficiently label DNA regions of $10 \mathrm{~kb}$ or less; therefore, it does not have the resolution to detect positional changes of single genes. In addition to FISH, the Lac operator/Lac repressor reporter system ${ }^{14}$ has also been used to visualize chromatin dynamics in transgenic plant lines ${ }^{15-17}$. However, because inserting a reporter construct into a specific locus in Arabidopsis remains challenging ${ }^{18}$, and because tandem repeat sequences tend to cluster together and thus could potentially alter the endogenous chromosomal organization at the transgene locus in Arabidopsis ${ }^{19}$, this reporter system has not been particularly useful to study the chromatin dynamics of specific endogenous loci.

Here we present a new protocol to label individual endogenous gene loci in Arabidopsis nuclei by using rolling-circle amplification of gene-specific padlock probes coupled with FISH. Using this new approach, we demonstrate that light triggers a rapid repositioning of the light-inducible $C A B$ locus from the nuclear interior to the nuclear periphery during its transcriptional activation. $C A B$ repositioning is regulated by the red (R)/far-red (FR) photoreceptors phytochromes (PHYs) and PHY-signalling components. In addition, $C A B$ repositioning appears to be a separate regulatory step occurring before its full transcriptional activation. Moreover, the light-inducible loci RBCS (Rubisco small subunit), PC (Plastocyanin) and GUN5 (Genomes uncoupled 5) undergo similar repositioning behaviour on their activation. Our data support a light-dependent gene regulatory mechanism in which PHYs activate light-inducible loci by relocating them to the nuclear periphery.

\section{Results}

Labelling of individual genes using padlock FISH. We developed a protocol to label individual gene loci in plant nuclei based on an in situ genotyping method for human cells established by
Nilsson and coworkers ${ }^{20}$. This method uses rolling-circle amplification of gene-specific circularizable oligonucleotides'padlock' probes-coupled with FISH (Fig. 1a and see Methods). To demonstrate that the padlock FISH protocol works for Arabidopsis nuclei, we first tested whether we could use the protocol to detect the centromeric 180 -bp repeats ${ }^{21}$. We designed a padlock probe recognizing the $30-\mathrm{bp}$ centromere sequence immediately flanking the HindIII site (Fig. 1a and Supplementary Table S1). Then, we tested whether the 180-bp repeat signals from padlock FISH were co-localized with immunolocalization signals for the centromeres, which were labelled using anti-HTR12 antibodies against CENH3 (ref. 22). As shown in Fig. 1b, 92.9\% of the 180-bp FISH signals $(n=435)$ were at least partially overlapping with a CENH3 signal, suggesting that the padlock FISH protocol can effectively label the 180 -bp repeats. To show that the protocol also works for a single-gene locus, we chose the UBQ11 (At4g05050) locus. Because UBQ11 lies within the pericentromeric region on chromosome 4 (ref. 23), we expected that the UBQ11 locus should also be associated with a centromere. Indeed, the UBQ11 padlock FISH signals were always closely associated with a CENH3 signal (Fig. 1c); the average distance from the UBQ11 signal to the closest CENH3 signal was $0.37 \pm 0.13 \mu \mathrm{m}(n=30$, mean \pm s.d. $)$. Together, these results demonstrate that in principle, the padlock FISH protocol can be used to determine the position of both repetitive sequences and single-gene loci in plant nuclei.

Light-dependent repositioning of the $C A B$ locus. To test whether light regulates the spatial organization of individual genes, we turned to some of the best-characterized light-induced genes, the $C A B$ genes. Three members of the $C A B$ gene family, $C A B 1-3$ (Atlg29930, Atlg29920 and Atlg29910), are clustered in a 7-kb region on chromosome 1 , hereafter referred to as the $C A B$ locus (Supplementary Fig. S1). In mesophyll cells, all three $C A B$ genes are repressed in the dark and are rapidly induced by light via the $\mathrm{R}$ and FR photoreceptors $\mathrm{PHY} \mathrm{s}^{24,25}$. To study $C A B$ positioning specifically in the mesophyll nuclei, we first characterized the nuclear morphology of various cell types and defined the criteria to identify mesophyll cell nuclei among isolated nuclei (Supplementary Fig. S2 and Supplementary Discussion). We then asked whether the $C A B$ locus is found at distinct subnuclear positions between the inactive state in the dark (D) and the active state in the light. Using a padlock probe specific to the CAB1-3 genes (Supplementary Fig. S1 and Supplementary Table S1), we found a dramatic difference in the radial distribution of the $C A B$ locus in mesophyll nuclei between $\mathrm{D}$ and monochromatic $\mathrm{R}$ or FR light conditions (Fig. 2a). In the dark, when the $C A B$ genes are repressed, the $C A B$ locus was localized in the nuclear interior (Fig. 2a). In striking contrast, in $\mathrm{R}$ or FR light, when the genes were activated, the distribution of $C A B$ loci peaked near the nuclear periphery; this phenomenon was more pronounced in $\mathrm{R}$ light than in FR light (Fig. 2a). We arbitrarily defined the area within $0.2 \mu \mathrm{m}$ of the nuclear edge as the 'nuclear peripheral zone' and calculated the percentage of $C A B$ loci localized to the nuclear peripheral zone with s.e. from two or three biological replicates in each condition. The percentage of $C A B$ loci localized to the nuclear peripheral zone increased drastically from $21 \pm 3 \%$ $(n=140)$ in $\mathrm{D}$ to $35 \pm 3 \%(n=124)$ in FR light and $49 \pm 4 \%$ $(n=153)$ in $\mathrm{R}$ light (Fig. 2a). This increase in the percentage of $C A B$ loci at the nuclear periphery correlates with an increase in the expression of $C A B$ in FR and $\mathrm{R}$ compared with $\mathrm{D}$ (Fig. 2b). In contrast, the distribution of a light-independent control gene locus, PP2A (protein phosphatase 2A, At1g69960), was not altered by light (Supplementary Fig. S3). Together, these results indicate that the $C A B$ locus is repositioned from the nuclear 
a
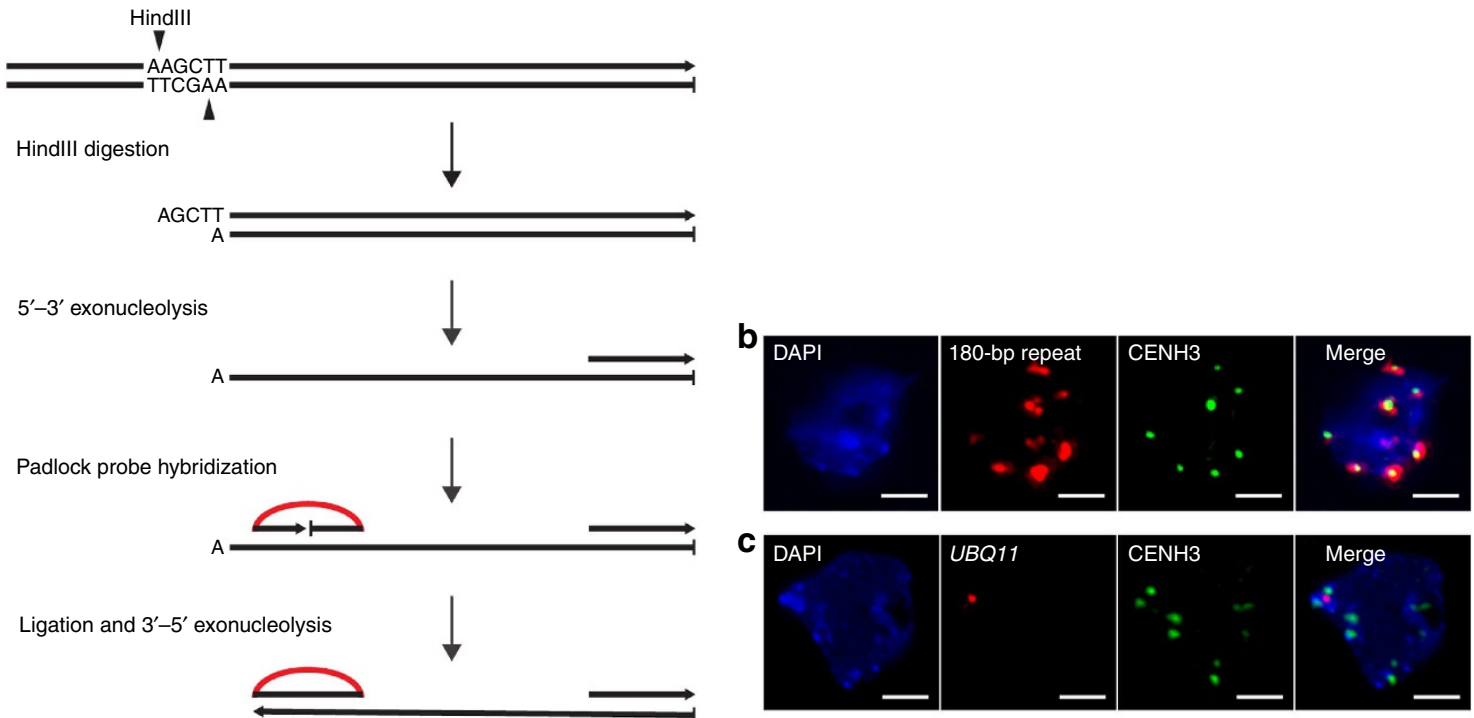

Ligation and $3^{\prime}-5^{\prime}$ exonucleolysis

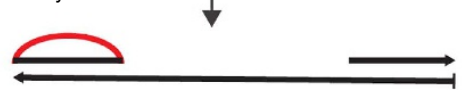

Rolling-circle amplification and FISH

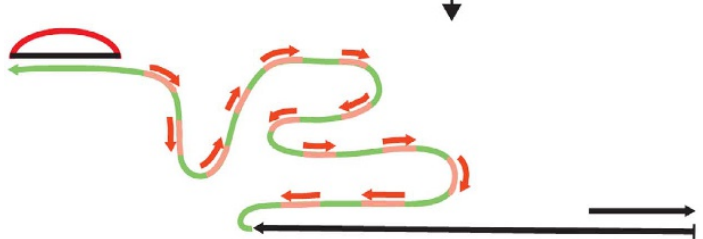

Figure 1 | Labelling of centromeric 180-bp satellite repeats and the UBQ11 locus using padlock FISH. (a) Schematic illustration of the padlock FISH protocol using the 180-bp repeat probe as a model. The illustration is modified from figure 1 of Larsson et al..$^{20}$ First, the target DNA is digested by HindllI (see the list of enzymes used for other padlock probes used in this study in Supplementary Table S1) and subsequently by lambda $5^{\prime}-3^{\prime}$ exonuclease to generate single-stranded DNA flanking the HindlII site. The padlock probes, which contain target sequences near the Hindlll site and a 'detection probe' sequence (shown in red, see Supplementary Table S1), are annealed to the target DNA and the ends of the probes are joined by ligation. Then, phi29 DNA polymerase is added. The $3^{\prime}-5^{\prime}$ exonuclease activity of the polymerase removes the $3^{\prime}$-protruding sequence beyond the padlock probe, and then the polymerase uses the circularized probe as the template and amplifies its DNA by rolling-circle amplification. The amplified DNA product is detected by hybridization of fluorescent-labelled oligonucleotides (shown in red, see 'detection probes' in Supplementary Table S1). Arrowheads indicate the 3' ends, and bars indicate the $5^{\prime}$ ends. (b) Representative images showing the localization of centromeric 180-bp repeats (red) labelled by padlock FISH and centromeres (green) by immunolocalization using anti-HTR12 antibodies against CENH3 in nuclei from cotyledons of 4-day-old Col-0 seedlings grown in R light. The nuclei were counterstained with DAPI (blue). (c) Representative images showing the localization of the UBQ11 locus (red) labelled by padlock FISH and centromeres (green) by immunolocalization using anti-HTR12 antibodies in nuclei from cotyledons of 4-day-old Col-0 seedlings grown in R light. The nuclei were counterstained with DAPI (blue). Scale bars, $2 \mu \mathrm{m}$.

interior to the nuclear periphery during transcriptional activation by $\mathrm{R}$ and FR light.

We then measured the kinetics of $C A B$ repositioning when dark-grown seedlings were first exposed to light (Fig. 2c,d). The percentage of $C A B$ loci at the nuclear periphery was rapidly increased from $21 \pm 3 \%$ in D to $49 \pm 1 \%(n=170$ mean \pm s.e. of three independent replicates, two-sample $t$-test, $P<0.001)$ within $3 \mathrm{~h}$ of $\mathrm{R}$ light exposure (Fig. 2d). These data further show that on light induction, the $C A B$ locus is rapidly relocated from the nuclear interior to the nuclear periphery. Again, the expression level of the $C A B$ genes correlated closely with the percentage of $C A B$ loci at the nuclear periphery (Fig. $2 \mathrm{c}, \mathrm{d}$ ), further supporting the notion that the repositioning of the $C A B$ locus to the nuclear periphery is associated with its transcriptional activation. Interestingly, although it took only $3 \mathrm{~h}$ for the percentage of peripherally localized $C A B$ loci to reach the same level as that in continuous R light (Fig. 2d), the expression level of the $C A B$ genes was still far below the fully activated level in continuous $\mathrm{R}$ light (Fig. 2c). These data suggest that $C A B$ repositioning is a separate regulatory step that occurs before its full transcriptional activation.
$C A B$ repositioning is triggered by $\mathrm{PHYs}$. It has been shown that the activation of $C A B$ expression is dependent on the $\mathrm{R}$ and FR photoreceptors, the PHYs. In Arabidopsis, PHYA and PHYB are the prominent photoreceptors in sensing continuous monochromatic FR and $\mathrm{R}$ light, respectively; $C A B$ activation is almost completely abolished in the phyA-211 mutant in continuous FR light and in the phyB-9 mutant in continuous $\mathrm{R}$ light (Fig. 3a,b) ${ }^{25,26}$. To test whether $C A B$ repositioning is also $\mathrm{PHY}$ dependent, we examined $C A B$ positioning in the $p h y A-211$ and phy $B$-9 mutants. In agreement with the expression data, the $C A B$ repositioning response was also substantially diminished in phyA211 in FR and in phyB-9 in R light (Fig. 3c,d), indicating that the light-dependent $C A B$ repositioning response is mainly dependent on PHYA in continuous FR light and on PHYB in continuous $\mathrm{R}$ light. Consistent with this notion, YHBox, a line expressing a constitutively active PHYB mutant ${ }^{27}$, was able to activate $C A B$ expression and trigger the repositioning of the $C A B$ locus to the nuclear periphery in the dark (Fig. 3e,f). On the basis of these results, we conclude that light-dependent $C A B$ repositioning to the nuclear periphery is mediated by the photoactivation of PHYs. 

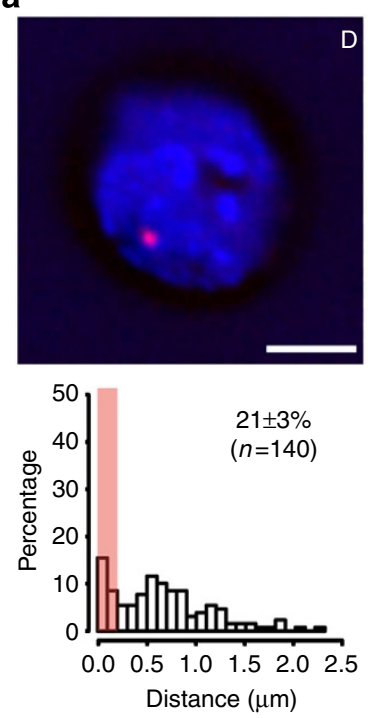

b

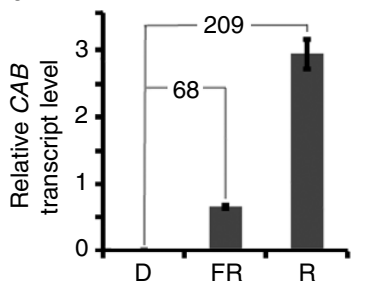

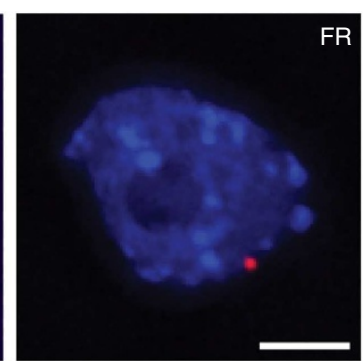

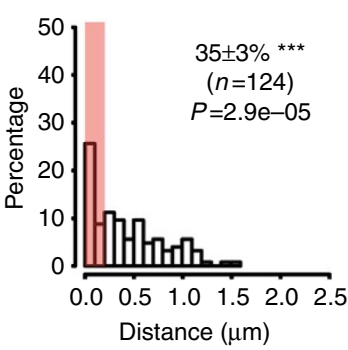

C

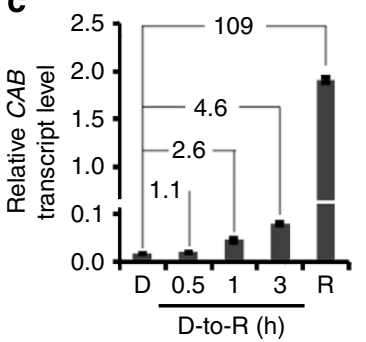

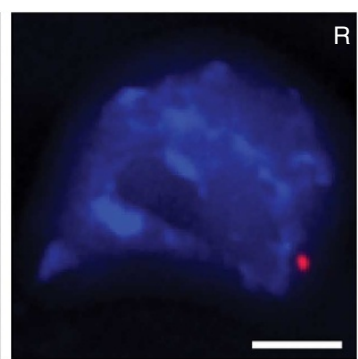

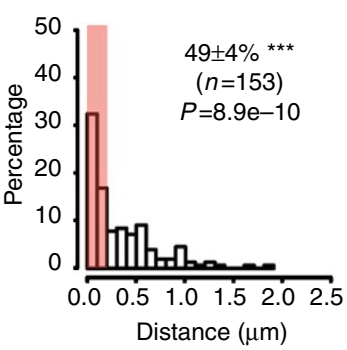

d

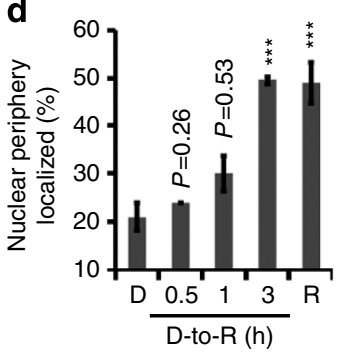

Figure 2 | Repositioning of the CAB locus from the nuclear interior to the nuclear periphery during light activation. (a) Representative images of $C A B$ positioning (top) and distribution of the $C A B$ locus (bottom) relative to the nuclear edge in mesophyll cell nuclei from 4-day-old Col-0 seedlings grown in the dark (D), far-red (FR), and red (R) light. The CAB locus was labelled using padlock FISH (red) and the nuclei were counterstained with DAPI

(blue). Scale bars, $2 \mu \mathrm{m}$ (in the images). The pink areas in the $C A B$ distribution panels represent the nuclear peripheral zone-the region $0.2 \mu \mathrm{m}$ or closer to the nuclear edge-and the average percentage of $C A B$ loci within the nuclear peripheral zone with s.e. from three independent replicates is shown in each panel. ' $n$ ' represents the total number of padlock FISH signals analysed from all replicates. The CAB distribution data from the R and FR samples were compared with that of the $D$ sample using Welch's two-sample $t$-test, ${ }^{\star \star \star} P<0.001$. (b) qRT-PCR analysis of CAB1-3 messenger RNA levels from seedlings grown in $\mathrm{D}$, FR or $\mathrm{R}$ light. Transcript levels were calculated relative to those of $P P 2 A$. Fold-changes of $C A B$ expression in $\mathrm{R}$ or $\mathrm{FR}$ relative to $\mathrm{D}$ conditions are shown. Error bars represent s.d. from three replicates. (c) Relative expression levels of $C A B$ s during the $D$-to-R transition. Fold-changes of $C A B$ expression in R-treated samples relative to that in the dark are shown. Error bars represent s.d. from three replicates. (d) Average percentage of $C A B$ loci at the nuclear periphery at the indicated time points during the $D$-to-R transition. Error bars in panel $\mathbf{d}$ represent s.e. calculated from three independent replicates (except for the 0.5 -h time point, which had two replicates). The $C A B$ distribution data from the $D$-to- $R$ transition samples were compared with that of the $D$ sample using Welch's two-sample $t$-test, ${ }^{\star \star \star} P<0.001$.

$C A B$ positioning is regulated by COP1, DET1 and PIFs. To further confirm the role of $\mathrm{PHY}$ signalling in $C A B$ positioning, we asked whether $C A B$ positioning is regulated by known PHYsignalling components. PHY signalling has been extensively investigated, and many signalling components mediating the induction of $C A B$ expression have been identified ${ }^{3,4}$. Among the positive factors involved in $C A B$ expression is the master bZIP transcriptional regulator HY5 (elongated HYpocotyl 5), which is not essential for $C A B$ induction but binds directly to a G-box in the promoter of the $C A B$ genes and plays a role in the full activation of $C A B$ expression in the light (Fig. 4a) ${ }^{28-31}$. Interestingly, in $\mathrm{R}$ light, $C A B$ positioning in the hy5 mutant was statistically similar to that of Col-0 (Fig. 4b), indicating that HY5 does not play a major role in $C A B$ repositioning. These data also imply that $C A B$ repositioning and full activation could be regulated by distinct trans-factors.

A number of master repressors of PHY signalling have also been described, including DET1 (De-ETiolated 1$)^{32}$, COP1 (COnstitutively Photomorphogenic) ${ }^{33}$ and phytochrome interacting factors (PIFs) ${ }^{34}$. PIFs are master bHLH transcription factors that antagonize PHY-mediated light responses ${ }^{34}$; COP1 and DET1 are substrate recognition subunits of CULLIN4 E3 ubiquitin ligase complexes ${ }^{35,36}$, which determine the abundance of key transcription factors including HY5 and the PIFs ${ }^{37,38}$. DET1 has also been implicated in chromatin modification ${ }^{39}$. Knocking out DET1, COP1 or 4 PIF family members leads to constitutive photomorphogenetic responses in the dark, including the derepression of the $C A B$ genes (Fig. $4 \mathrm{c})^{2,32,33}$. We therefore examined $C A B$ positioning in dark-grown $\operatorname{det} 1-1, \operatorname{cop} 1-4$ and pifq mutants and asked whether the light-independent $C A B$ activation in these mutants is accompanied by $C A B$ localization to the nuclear periphery. As shown in Fig. $4 \mathrm{~d}$, the distribution of the $C A B$ locus in det1-1, cop1-4 and pifq in the dark was significantly different from that of dark-grown Col-0 and instead resembled that of Col-0 in R light. These results indicate that COP1, DET1 and the PIFs are essential for retaining the $C A B$ locus in the nuclear interior in the dark. Interestingly, although cop1-4 and det1-1 showed similar $C A B$-positioning patterns as pifq, $C A B$ expression levels in cop 1-4 and det1-1 were considerably lower than that in pifq (Fig. $4 \mathrm{c}, \mathrm{d}$ ). These data are consistent with 


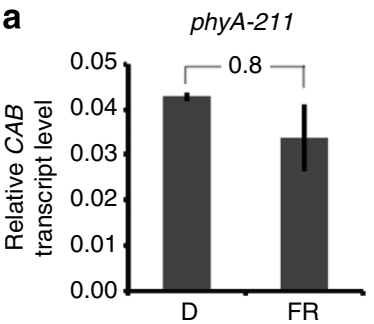

C
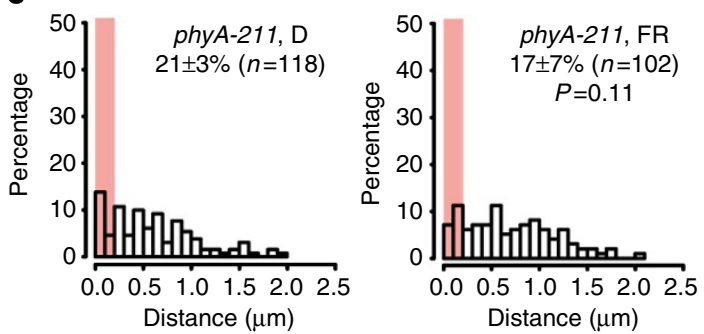

b

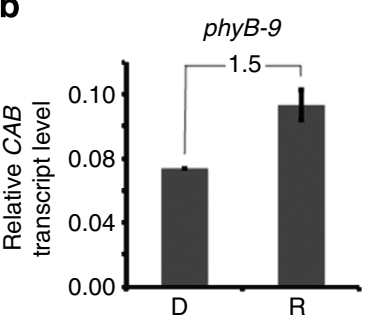

d
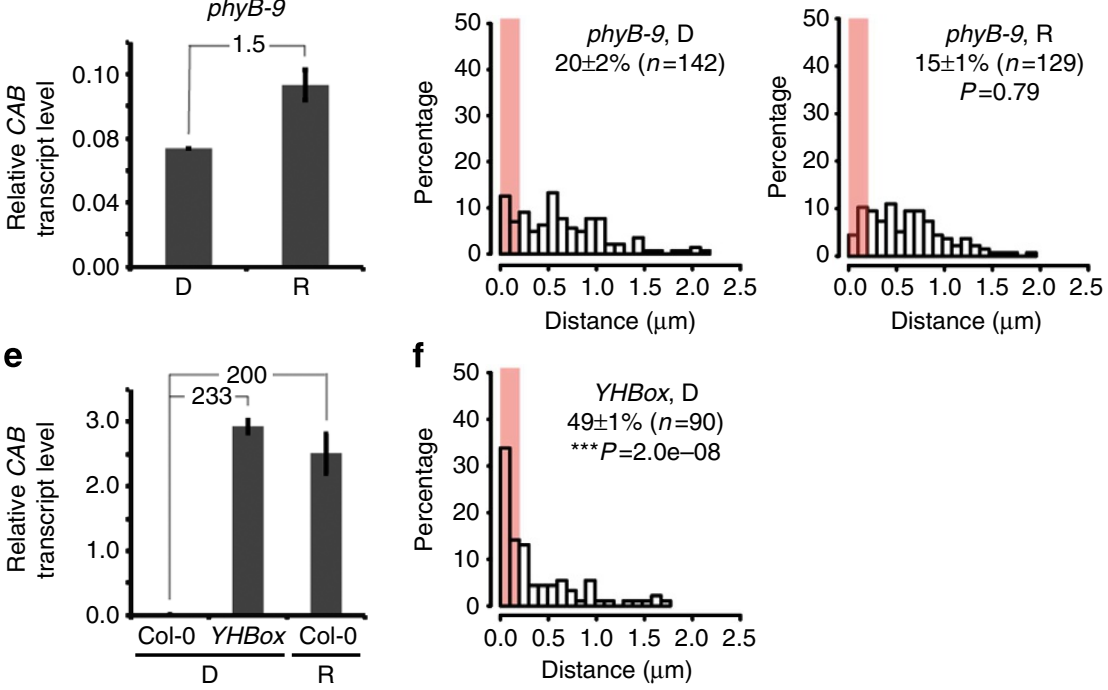

Figure 3 | CAB repositioning during light activation is PHY dependent. (a) qRT-PCR data showing the CAB-3 mRNA levels in 4-day-old phyA-211 seedlings grown in the D or FR light conditions. Transcript levels were calculated relative to those of $P P 2 A$. Fold-change of $C A B$ expression in FR relative to $D$ is shown. Error bars represent s.d. from three replicates. (b) qRT-PCR data showing the $C A B^{1-3}$ mRNA levels in 4-day-old phyB-9 seedlings grown in $D$ or $\mathrm{R}$ light conditions. Transcript levels were calculated relative to those of $P P 2 A$. Fold-change of $C A B$ expression in $\mathrm{R}$ relative to $\mathrm{D}$ is shown. Error bars represent s.d. from three replicates. (c) The distribution of $C A B$ loci and the average percentages of $C A B$ loci localized to the nuclear peripheral zone in phy $A-211$ in either the $D$ or FR light conditions. (d) The distribution of $C A B$ loci and the average percentages of $C A B$ loci localized to the nuclear peripheral zone in phyB-9 in either the D or R light conditions. (e) qRT-PCR data showing the CAB ${ }^{1-3}$ mRNA levels in 4-day-old Col-O and $Y H B o x$ seedlings grown in the D and Col-0 seedlings in $\mathrm{R}$ light. Transcript levels were calculated relative to those of PP2A. Fold-change of CAB expression in D-grown YHBox or R-grown Col-O relative to that of D-grown Col-O is shown. Error bars represent s.d. from three replicates. (f) The distribution of $C A B$ loci and the average percentages of $C A B$ loci localized to the nuclear peripheral zone in YHBox seedlings grown in the $D$. The $C A B$ distribution data in $\mathbf{c}, \mathbf{d}$ and $\mathbf{f}$ were from two independent replicates, and ' $n$ ' represents the total number of padlock FISH signals analysed from all replicates. Statistical analyses between FR and D samples of phyA-211, between R and D samples of phyB-9 and between D-grown YHBox and Col-0 were performed using Welch's two-sample t-test, and ${ }^{\star \star \star} P<0.001$.

previous reports that COP1 and DET1, but not the PIFs, also play a positive role in the full activation of $C A B$ expression in the light ${ }^{28,33}$. Our results provide a possible explanation for this phenomenon: COP1 and DET1 play opposing roles in repressing $C A B$ repositioning and promoting full $C A B$ activation, whereas PIFs are involved only in the repression of $C A B$ positioning to the nuclear periphery. Together, these results further support the conclusion that $C A B$ positioning is regulated by $\mathrm{PHY}$ signalling and begin to elucidate distinct roles of PHY signalling components in $C A B$ positioning.

Light-dependent repositioning of other light-inducible loci. To test whether repositioning to the nuclear periphery is a common phenomenon among light-induced genes, we examined three additional light-induced photosynthesis-related genes, RBCS1A (At1g67090), GUN5 (At5g13630) and PC (At1g76100) (Fig. 5a-c), which are distributed throughout the Arabidopsis genome. As shown in Fig. 5d-f, all three loci were localized to the nuclear interior in the dark and to the nuclear periphery in the light, when they are transcriptionally activated. These results support the notion that relocating genes from the nuclear interior to the nuclear periphery is a common mechanism of gene activation by light.

\section{Discussion}

We have demonstrated that light triggers the repositioning of a group of light-inducible genes from the nuclear interior to the nuclear periphery during their transcriptional activation. Our experimental evidence supports a model in which PHY signalling regulates two separate regulatory steps during $C A B$ induction: its repositioning and its full activation (Fig. 6). This conclusion is supported by three lines of evidence: $C A B$ repositioning to the nuclear periphery occurs before its full activation; the percentage of $C A B$ loci at the nuclear periphery correlates closely with $C A B$ 's expression level; and $C A B$ repositioning and its full transcriptional activation are regulated by distinct trans-factors. Although it is still not clear whether $C A B$ repositioning is required for its full activation, our data suggest that the repositioning of individual genes to the nuclear periphery is an important regulatory step in light-regulated gene induction (Fig. 6). 


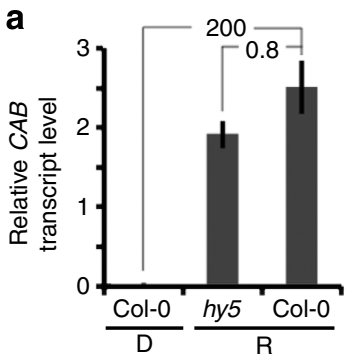

b

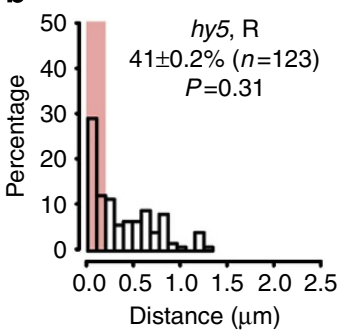

d

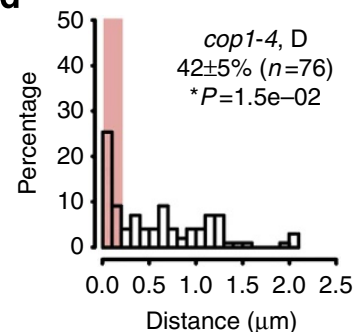

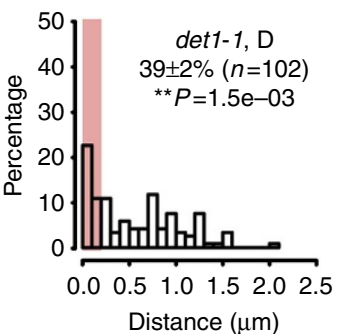

C
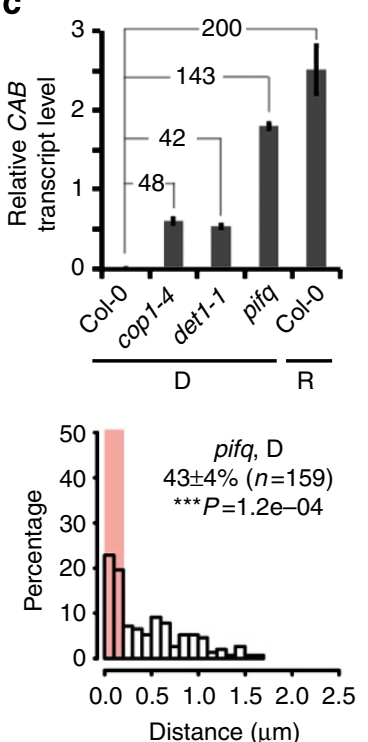

Figure 4 | The CAB repositioning response in photomorphogenetic mutants. DET1, COP1 and PIFs, but not HY5, are involved in the regulation of $\mathbf{C A B}$ positioning in Arabidopsis. (a) Relative expression level of $C A B s$ in $\mathrm{Col}-0$ and hy 5 in $\mathrm{R}$ light and Col- 0 in the $\mathrm{D}$. Fold-changes of $C A B$ expression between R-grown and D-grown Col-0 and between R-grown hy5 and Col-0 are shown. (b) Distribution of CAB loci and the average percentages of nuclear peripheral localized $C A B$ loci in the hy 5 mutant grown in $R$ light. Data were from two independent replicates, and ' $n$ ' represents the total number of padlock FISH signals analysed from both replicates. Statistical analyses using Welch's two-sample $t$-test showed no significant difference between the $C A B$ distributions in hy5 and Col- 0 in $\mathrm{R}$ light $(P=0.31)$. (c) The relative expression level of $C A B s$ in cop1-4, det1-1 and pifq in $\mathrm{D}$. Fold-changes of $C A B$ expression between the respective mutant and Col-O are shown. (d) Distribution of $C A B$ loci and the average percentage of $C A B$ loci at the nuclear periphery in D-grown cop1-4, det1-1 and pifq. The CAB distribution data were from at least two independent replicates, and ' $n$ ' represents the total number of padlock FISH signals analysed from independent replicates. Statistical analyses between $C A B$ distribution data from each mutant and corresponding Col-0 control samples were performed using Welch's two-sample $t$-test; ${ }^{\star} P<0.05,{ }^{\star \star} P<0.01$ and ${ }^{\star \star \star} P<0.001$. Error bars in panels $\mathbf{a}$ and $\mathbf{c}$ represent s.d. from three replicates.

Our study also provides key initial evidence supporting the biological significance of gene positioning in transcriptional regulation in the plant kingdom. The gene repositioning phenomenon of the light-induced genes examined here resembles the behaviour of a number of inducible genes in yeast, including INO1, GAL1, GAL2, HSP104 and SUC2, which relocate from the nucleoplasm to the nuclear periphery on activation ${ }^{40-43}$. Therefore, our results suggest that the basic principles of spatial organization of genes during transcriptional regulation might be part of an ancient regulatory mechanism that evolved before the divergence of the fungal/animal and plant lineages $\sim 1.6$ billon years ago ${ }^{8}$.

Our findings that PHY-signalling components play distinct roles in $C A B$ repositioning provide insight into the signalling pathways as well as the molecular mechanisms that regulate gene positioning in plants. Although many studies from yeast and metazoan models have shown the implications of gene repositioning in transcription, the molecular mechanisms of gene repositioning are still largely unknown. Studies of the INO1 gene in yeast have provided the most in-depth mechanism so far: gene positioning could be controlled by both cis-regulatory elements and trans-factors ${ }^{44-46}$. Our results suggest that the PIFs are potential trans-factors that retain light-inducible loci in the nuclear interior in the dark (Fig. 6). A possible mechanism is that PIFs induce gene-silencing histone modifications at the target loci. Consistent with this hypothesis, a recent study showed that PIF3 directly binds to the G-box elements of light-inducible photosynthetic genes, including GUN5 and the $C A B$ paralogue LHCB2.1, and it promotes gene silencing by recruiting the histone deacetylase HDA15 (ref. 47). Further supporting this model, histone deacetylase 6, along with PHYB, is involved in lightdependent chromatin compaction in Arabidopsis ${ }^{48}$. DET1 could be another trans-factor that retains light-inducible genes in the nucleoplasm. Tomato DET1 binds to the nonacetylated aminoterminal tail of histone $\mathrm{H}_{2} \mathrm{~B}^{39}$; in addition, DET1 has been shown to act as a key transcriptional repressor ${ }^{49}$. Therefore, DET1 could work in concert with PIFs to retain light-inducible genes in the nuclear interior. $C A B$ repositioning by light is probably triggered by the PHY-dependent degradation of $\mathrm{PIFs}^{34}$. Although the mechanism of PIF degradation is still largely unknown, COP1 has been shown to be required for PIF3 accumulation in the dark ${ }^{38}$. Therefore, both PHYs and COP1 might regulate gene positioning by controlling PIF abundance (Fig. 6). Previous studies in yeast and metazoan systems also indicate that the nuclear pore complex plays an important role in promoting gene activation $^{35-37}$. It remains to be investigated whether lightinduced gene loci are directly associated with the nuclear pore complex in plants.

All of the photosynthesis-regulated genes analysed here belong to a group of light-regulated genes that are induced during the dark-to-light transition and remain induced in the light. Although all four of these genes showed similar light-dependent repositioning behaviour, it is still far from sufficient to conclude that all light-induced genes relocate to the nuclear periphery in the light. It would not be surprising if different mechanisms are involved in the activation of different classes of light-inducible genes $^{2}$. It would be interesting to examine the group of early light-induced genes ${ }^{2}$ that are transiently induced by light during the dark-to-light transition and see whether these genes are only temporarily localized to the nuclear periphery during their activation. It also remains to be investigated whether lightrepressed genes ${ }^{2}$ are activated at the nuclear periphery in the dark. In addition, it is still not clear whether repositioning to the nuclear periphery is also involved in gene activation in other 
a

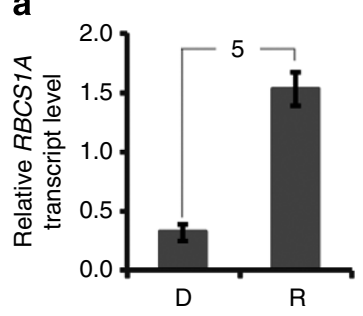

b

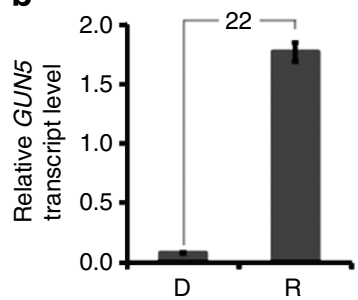

C

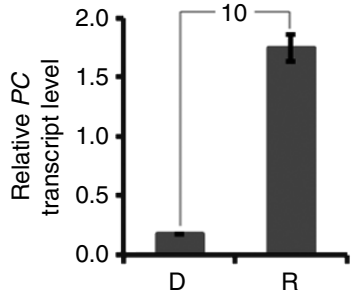

d

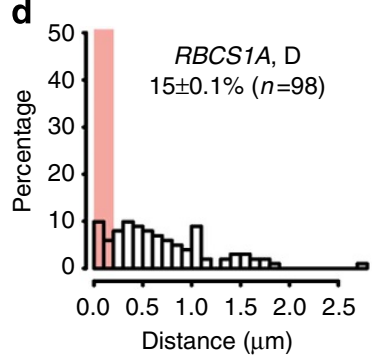

e

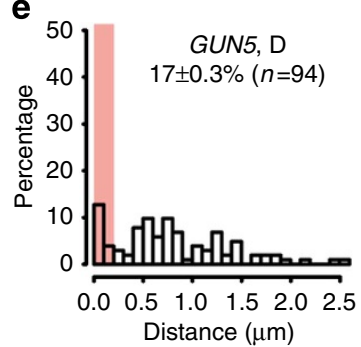

f

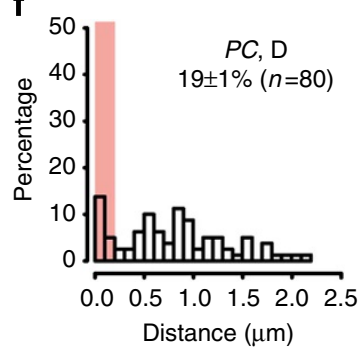

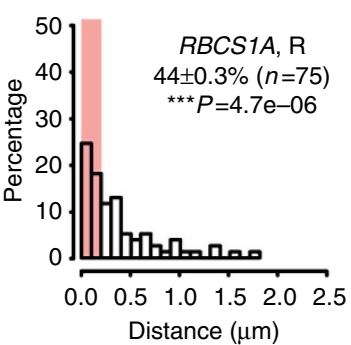

GUN5, R
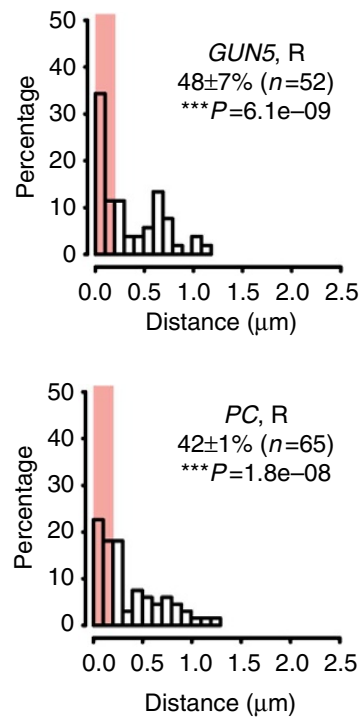

Figure 5 | Light-dependent repositioning of other light-inducible loci. (a) qRT-PCR results showing the relative expression levels of RBCSTA in 4-day-old Col-0 seedlings grown either in the $\mathrm{D}$ or $\mathrm{R}$ light conditions. Transcript levels were calculated relative to those of PP2A. (b) qRT-PCR results showing the relative expression levels of GUN5 in 4-day-old Col-0 seedlings grown either in the D or R light conditions. Transcript levels were calculated relative to those of PP2A. (c) qRT-PCR results showing the relative expression levels of $P C$ in 4-day-old Col-0 seedlings grown either in the $\mathrm{D}$ or $\mathrm{R}$ light conditions. Transcript levels were calculated relative to those of $P P 2 A$. Error bars in panels $\mathbf{a}, \mathbf{b}$ and $\mathbf{c}$ represent s.d. from three replicates. (d) The distribution and the average percentage of peripherally localized RBCS1A loci in $\mathrm{D}$ and $\mathrm{R}$ conditions. (e) The distribution and the average percentage of peripherally localized GUN5 loci in D and R conditions. (f) The distribution and the average percentage of peripherally localized $P C$ loci in D and R conditions. The gene distribution data in $\mathbf{d}, \mathbf{e}$ and $\mathbf{f}$ were from two independent replicates, and ' $n$ ' represents the total number of padlock FISH signals analysed from all replicates. Statistical analyses between R and D samples for each gene were performed using Welch's two-sample $t$-test, ${ }^{\star * \star} P<0.001$.

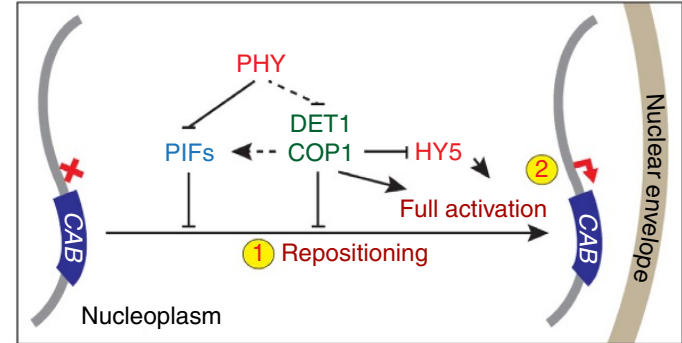

Figure 6 | Model for $C A B$ repositioning and gene activation during its induction by light. The transcriptionally silenced $C A B$ locus is localized to the nuclear interior. Light activates PHY signalling to trigger the $C A B$ locus' repositioning to the nuclear periphery and its full transcriptional activation. Although it is still not clear whether $C A B$ localization to the nuclear periphery directly causes its activation, $C A B^{\prime}$ s repositioning and full activation appear to be separate steps. $C A B$ localization to the nuclear periphery is inhibited by COP1, DET1 and PIFs, whose functions are repressed by $\mathrm{PHYS}$ in the light. COP1 could inhibit $C A B$ repositioning by stabilizing at least one of the PIFs, PIF3. Both DET1 and COP1 play separate and positive roles in the full activation step, which also requires HY5.

NATURE COMMUNICATIONS | 5:3027 | DOI: 10.1038/ncomms4027 | www.nature.com/naturecommunications signalling pathways in plants. On the basis of the principles learned from yeast and metazoan models, there are different gene activating and silencing domains in the nucleus ${ }^{5-7}$; therefore, we anticipate that more functional domains will also be defined in plant nuclei. A recent elegant study on the localization of FLC alleles suggests that gene repositioning is also involved in gene silencing in Arabidopsis ${ }^{17}$. We expect that our study on lightregulated gene repositioning and the padlock FISH method described here will stimulate further studies on genome organization in plants.

\section{Methods}

Plant materials and growth conditions. Wild-type Col-0, phyA-211 (ref. 50), phyB-9 (ref. 51), hy5 (ref. 52), cop1-4 (ref. 33), det1-1 (ref. 32) and pifq (ref. 53) were used to characterize $C A B$ positioning. The YHBox line is a transgenic line carrying a 35S:YHB-YFP-FLAG construct in the phyB-9 mutant background; this line resembles the previously described constitutively active phyB allele, $Y H B^{54}$, but in the Col-0 background (Supplementary Fig. S4). Arabidopsis seed sterilization, stratification and growth conditions were described previously ${ }^{55}$.

Padlock FISH. Plant fixation and isolation of nuclei were performed based on Schubert et al. ${ }^{56}$, and the padlock FISH procedure was developed based on Larsson et $a l^{20}$ with modifications. Seedlings grown for $100 \mathrm{~h}$ under the indicated 
conditions were fixed in cold fixation buffer ( $4 \%$ formaldehyde, $10 \mathrm{mM}$ Tris- $\mathrm{HCl}$ $\mathrm{pH} 7.5,10 \mathrm{mM}$ EDTA, $100 \mathrm{mM} \mathrm{NaCl}$ ) for 30 min under vacuum. Approximately 100 cotyledons were chopped in $50 \mu$ lysis buffer $(15 \mathrm{mM}$ Tris- $\mathrm{HCl}$ pH 7.5, $2 \mathrm{mM}$ EDTA, $0.5 \mathrm{mM}$ spermine- $4 \mathrm{HCl}, 80 \mathrm{mM} \mathrm{KCl}, 20 \mathrm{mM} \mathrm{NaCl}$ and $0.1 \%$ Triton X-100) with a razor blade on a slide. The resulting suspension containing the released nuclei was transferred into four volumes of nuclei suspension buffer $(100 \mathrm{mM}$ Tris- $\mathrm{HCl} \mathrm{pH} \mathrm{7.5,} 50 \mathrm{mM} \mathrm{KCl}, 2 \mathrm{mM} \mathrm{MgCl} 2,5 \%$ sucrose and $0.05 \%$ Tween-20). The nuclear suspension was spotted on a slide and air-dried overnight at room temperature. The slides were either used immediately or stored at $-20^{\circ} \mathrm{C}$.

All subsequent steps were performed in a $55-\mu$ l SecureSeal chamber (Grace Bio-Labs, Bend, OR) and are illustrated in Fig. 1a. The nuclei were washed in PBS and permeabilized with PBS containing $0.2 \%$ Triton X-100. After two washes in PBS, the slides were incubated with a DNA digestion solution containing $0.5 \mathrm{U}^{-1}$ of restriction enzyme (New England Biolabs, Ipswich, MA) at $37^{\circ} \mathrm{C}$ for $30 \mathrm{~min}$; the specific restriction enzymes used for each target gene are listed in Supplementary Table S1. After two washes in buffer A (100 mM Tris- $\mathrm{HCl} \mathrm{pH} \mathrm{7.5,}$ $150 \mathrm{mM} \mathrm{NaCl}$ and $0.05 \%$ Tween-20), the slides were treated with $0.2 \mathrm{U} \mathrm{\mu l}{ }^{-1}$ of $5^{\prime}-$ $3^{\prime}$ Lambda exonuclease (New England Biolabs) in Lambda exonuclease buffer containing $0.2 \mu \mathrm{g}^{-1} \mathrm{BSA}$ and $10 \%$ glycerol at $37^{\circ} \mathrm{C}$ for $30 \mathrm{~min}$ to generate a single-stranded target sequence (Fig. 1a). Then, the slides were briefly washed twice in buffer A and incubated with T4 ligase buffer supplemented with $0.1 \mu \mathrm{M}$ genespecific padlock probes (Supplementary Table S1), $0.1 \mathrm{U} \mathrm{\mu l}^{-1}$ T4 ligase (New England Biolabs), $1 \mathrm{mM}$ ATP, $250 \mathrm{mM} \mathrm{NaCl}$ and $0.2 \mu \mathrm{g} \mu \mathrm{l}^{-1}$ BSA. The ligation reaction was carried out at $37^{\circ} \mathrm{C}$ for $30 \mathrm{~min}$, which allowed the padlock probes to anneal to the target sequence and to circularize (Fig. 1a). After one wash in buffer B $(2 \times$ saline-sodium citrate buffer and $0.05 \%$ Tween-20), the slides were incubated in $1{\mathrm{U} \mathrm{l}^{-1}}^{-1}$ of phi29 DNA polymerase (Fermentas, Burlington, Canada) in $1 \times$ phi29 buffer supplemented with $0.25 \mathrm{mM}$ dNTPs, $0.2 \mu \mathrm{g} \mu \mathrm{l}^{-1}$ BSA and $10 \%$ glycerol at $37^{\circ} \mathrm{C}$ for $2 \mathrm{~h}$. In this step, the padlock probe sequence was amplified by rolling-circle amplification (Fig. 1a). The slides were then incubated with $0.25 \mu \mathrm{M}$ fluorescently tagged detection oligonucleotide probes (Supplementary Table S1) in $2 \times$ saline-sodium citrate buffer containing $20 \%$ formamide at $37^{\circ} \mathrm{C}$ for $20 \mathrm{~min}$. Unbound fluorescent probes were washed away with five washes in PBS with $0.05 \%$ Tween at $37^{\circ} \mathrm{C}$ and three washes with PBS at room temperature. The nuclei were counterstained with $4^{\prime}, 6$-diamidino-2-phenylindole (DAPI; $500 \mathrm{ng} \mathrm{ml}^{-1}$ ) at room temperature for $20 \mathrm{~min}$, washed three times with water, mounted in Prolong Gold antifade reagent (Life Technologies, Carlsbad, CA) and sealed with fast-dry nail polish.

On the basis of our experiments on the $C A B$ and $P P 2 A$ loci, and assuming that all nuclei were diploid, the detection efficiency for each experiment ranged from 4 to $7 \%$ of all potential target sequences. This detection efficiency is slightly lower than the $10 \%$ detection efficiency reported for animal cells ${ }^{20}$. Given that some of the nuclei could be polyploid, the actual detection efficiency of this method is expected to be a slightly lower than this range. As a result, there was only one padlock FISH signal in the majority of nuclei examined.

Immunolocalization. Antibodies used were rabbit polyclonal antisera against HTR12 (1:100), recognizing Arabidopsis CENH3 (ref. 22), and mouse monoclonal antibodies against the nuclear pore complex (1:250, QE5, recognizing Nup214, Nup153 and p62 of the mammalian nuclear pore complex, Covance, Princeton, $\mathrm{NJ})^{57}$. Immunolocalization was performed as described previously ${ }^{58,59}$. For combined padlock FISH and immunolabeling, after the padlock FISH procedure, the slides were washed three times with PBS and blocked with PBS containing $3 \%$ BSA for $30 \mathrm{~min}$ at $37^{\circ} \mathrm{C}$. The slides were then incubated with primary antisera overnight at room temperature or at $37^{\circ} \mathrm{C}$. After five washes in PBS, the slides were incubated with secondary antibodies-either donkey-anti-mouse-AlexaFluor 488 or donkey-anti-rabbit-AlexaFluor 488 (Life Technologies, 1:300 dilution)-at $37^{\circ} \mathrm{C}$. The slides were washed five times in PBS and counterstained with $500 \mathrm{ng} \mathrm{ml}^{-1}$ DAPI.

Fluorescence microscopy. Mesophyll nuclei were selected based on their morphology as described in Supplementary Fig. S2. Three-dimensional image stacks with a $z$-step size of $0.2 \mu \mathrm{m}$ were acquired with a DeltaVision microscope system (Applied Precision, Issaquah, WA) consisting of an IX-71 Olympus inverted microscope (Olympus, Tokyo, Japan) with a UPLSAPO oil immersion objective lens $(\times 100$, numerical aperture 1.40; Olympus) and a Coolsnap HQ2 high-resolution charge-coupled device camera. DAPI, AlexaFluor 488, CY3 and CY5 were detected with appropriate filters. The image stacks were deconvolved with a constrained iterative of 15 by using softWoRx software (Applied Precision) on an Octane Workstation (SGI, Mountain View, CA).

Gene positioning measurements and data analysis. To measure the distance from the padlock FISH signal of a gene locus to the nuclear edge, an optical section with the strongest and most focused padlock FISH signal was used. The nuclear edge was determined by DAPI intensity as described in Supplementary Fig. S5a,b and outlined using the two-dimensional polygon finder function in softWoRx (Supplementary Fig. S5c,d). Then, using the centre of the padlock FISH signal as the centre, a circle was drawn whose circumference intersected the nuclear edge (Supplementary Fig. S5c). The distance between the intersection point and the center of the padlock FISH signal was defined as the distance between the gene locus and the nuclear edge. For each light condition or genotype, at least two biological replicates were analysed. The data from all replicates were combined and the radial distribution of each gene locus was plotted as a histogram with a bandwidth of $0.1 \mu \mathrm{m}$ in $\mathrm{R}$ ( ref. 60). The nuclear peripheral zone was defined as the area within $0.2 \mu \mathrm{m}$ of the nuclear edge. The percentage of the padlock FISH signals localized within the nuclear peripheral zone was calculated for each independent experiment, and the means of the percentages and the s.e. are shown in the figures. The normality of the data was tested in R with a Quantile-Quantile plot, and the statistical significance between gene distribution data was tested using Welch's two-sample $t$-test.

RNA extraction and qRT-PCR. RNA extraction and quantitative reverse transcriptase (RT)-PCR were performed as previously described ${ }^{61}$. Total RNA from seedlings of the indicated genotypes and growth conditions was isolated using the Spectrum Plant Total RNA Kit (Sigma, Saint Louis, MO). Complementary DNA was synthesized from messenger RNA using the Superscript II First-Strand cDNA Synthesis Kit (Invitrogen, Carlsbad, CA) according to the manufacturer's protocol. qRT-PCR was then performed with FastStart Universal SYBR Green Master Mix (Roche, Indianapolis, IN) in a Mastercycler ep Realplex thermal cycler (Eppendorf, Hauppauge, NY). Genes and primer sets used for qRT-PCR are listed in Supplementary Table S2.

\section{References}

1. Franklin, K. A. \& Quail, P. H. Phytochrome functions in Arabidopsis development. J. Exp. Bot. 61, 11-24 (2010).

2. Leivar, P. et al. Definition of early transcriptional circuitry involved in light-induced reversal of PIF-imposed repression of photomorphogenesis in young Arabidopsis seedlings. Plant Cell 21, 3535-3553 (2009).

3. Chen, M. \& Chory, J. Phytochrome signaling mechanisms and the control of plant development. Trends Cell. Biol. 21, 664-671 (2011).

4. Jiao, Y., Lau, O. S. \& Deng, X. W. Light-regulated transcriptional networks in higher plants. Nat. Rev. Genet. 8, 217-230 (2007).

5. Egecioglu, D. \& Brickner, J. H. Gene positioning and expression. Curr. Opin. Cell. Biol. 23, 338-345 (2011).

6. Chuang, C. H. \& Belmont, A. S. Moving chromatin within the interphase nucleus-controlled transitions? Semin. Cell Dev. Biol. 18, 698-706 (2007).

7. Takizawa, T., Meaburn, K. J. \& Misteli, T. The meaning of gene positioning. Cell 135, 9-13 (2008).

8. Wang, D. Y., Kumar, S. \& Hedges, S. B. Divergence time estimates for the early history of animal phyla and the origin of plants, animals and fungi. Proc. R. Soc. Lond. 266, 163-171 (1999).

9. Schubert, I. \& Shaw, P. Organization and dynamics of plant interphase chromosomes. Trends Plant Sci. 16, 273-281 (2011).

10. Fransz, P., De Jong, J. H., Lysak, M., Castiglione, M. R. \& Schubert, I. Interphase chromosomes in Arabidopsis are organized as well defined chromocenters from which euchromatin loops emanate. Proc. Natl Acad. Sci. USA 99, 14584-14589 (2002).

11. Pecinka, A. et al. Chromosome territory arrangement and homologous pairing in nuclei of Arabidopsis thaliana are predominantly random except for NORbearing chromosomes. Chromosoma 113, 258-269 (2004).

12. Lysak, M. A., Pecinka, A. \& Schubert, I. Recent progress in chromosome painting of Arabidopsis and related species. Chromosome Res. 11, 195-204 (2003).

13. Costa, S. \& Shaw, P. Chromatin organization and cell fate switch respond to positional information in Arabidopsis. Nature 439, 493-496 (2006).

14. Belmont, A. S., Li, G., Sudlow, G. \& Robinett, C. Visualization of large-scale chromatin structure and dynamics using the lac operator/lac repressor reporter system. Methods Cell Biol. 58, 203-222 (1999).

15. Rosin, F. M. et al. Genome-wide transposon tagging reveals location-dependent effects on transcription and chromatin organization in Arabidopsis. Plant J. 55, 514-525 (2008)

16. Matzke, A. J., Watanabe, K., van der Winden, J., Naumann, U. \& Matzke, M. High frequency, cell type-specific visualization of fluorescent-tagged genomic sites in interphase and mitotic cells of living Arabidopsis plants. Plant Methods 6, 2 (2010).

17. Rosa, S. et al. Physical clustering of FLC alleles during Polycomb-mediated epigenetic silencing in vernalization. Genes Dev. 27, 1845-1850 (2013).

18. Qi, Y. et al. Increasing frequencies of site-specific mutagenesis and gene targeting in Arabidopsis by manipulating DNA repair pathways. Genome Res. 23, 547-554 (2013).

19. Pecinka, A., Kato, N., Meister, A., Probst, A. V., Schubert, I. \& Lam, E. Tandem repetitive transgenes and fluorescent chromatin tags alter local interphase chromosome arrangement in Arabidopsis thaliana. J. Cell Sci. 118, 3751-3758 (2005).

20. Larsson, C. et al. In situ genotyping individual DNA molecules by targetprimed rolling-circle amplification of padlock probes. Nat. Methods 1, 227-232 (2004). 
21. Martinez-Zapater, J. M., Estelle, M. A. \& Somerville, C. R. A highly repeated DNA sequence in Arabidopsis thaliana. Mol. Gen. Genet. 204, 417-423 (1986).

22. Talbert, P. B., Masuelli, R., Tyagi, A. P., Comai, L. \& Henikoff, S. Centromeric localization and adaptive evolution of an Arabidopsis histone H3 variant. Plant Cell 14, 1053-1066 (2002).

23. Copenhaver, G. P. et al. Genetic definition and sequence analysis of Arabidopsis centromeres. Science 286, 2468-2474 (1999).

24. Karlin-Neumann, G. A., Sun, L. \& Tobin, E. M. Expression of light-harvesting chlorophyll a/b-protein genes is phytochrome-regulated in etiolated Arabidopsis thaliana seedlings. Plant Physiol. 88, 1323-1331 (1988).

25. Hamazato, F., Shinomura, T., Hanzawa, H., Chory, J. \& Furuya, M. Fluence and wavelength requirements for Arabidopsis $C A B$ gene induction by different phytochromes. Plant Physiol. 115, 1533-1540 (1997).

26. Barnes, S. A., Nishizawa, N. K., Quaggio, R. B., Whitelam, G. C. \& Chua, N. H. Far-red light blocks greening of Arabidopsis seedlings via a phytochrome A-mediated change in plastid development. Plant Cell 8, 601-615 (1996).

27. Hu, W., Su, Y. S. \& Lagarias, J. C. A light-independent allele of phytochrome B faithfully recapitulates photomorphogenic transcriptional networks. Mol. Plant 2, 166-182 (2009).

28. Maxwell, B. B., Andersson, C. R., Poole, D. S., Kay, S. A. \& Chory, J. HY5, Circadian Clock-Associated 1, and a cis-element, DET1 dark response element, mediate DET1 regulation of chlorophyll a/b-binding protein 2 expression. Plant Physiol. 133, 1565-1577 (2003).

29. Andronis, C., Barak, S., Knowles, S. M., Sugano, S. \& Tobin, E. M. The clock protein CCA1 and the bZIP transcription factor HY5 physically interact to regulate gene expression in Arabidopsis. Mol. Plant 1, 58-67 (2008).

30. Lee, J. et al. Analysis of transcription factor HY5 genomic binding sites revealed its hierarchical role in light regulation of development. Plant Cell 19, 731-749 (2007).

31. Zhang, H. et al. Genome-wide mapping of the HY5-mediated gene networks in Arabidopsis that involve both transcriptional and post-transcriptional regulation. Plant J. 65, 346-358 (2011).

32. Chory, J., Peto, C., Feinbaum, R., Pratt, L. \& Ausubel, F. Arabidopsis thaliana mutant that develops as a light-grown plant in the absence of light. Cell 58, 991-999 (1989)

33. Deng, X. W., Caspar, T. \& Quail, P. H. cop1: a regulatory locus involved in light-controlled development and gene expression in Arabidopsis. Genes Dev. 5, 1172-1182 (1991).

34. Leivar, P. \& Quail, P. H. PIFs: pivotal components in a cellular signaling hub. Trends Plant Sci. 16, 19-28 (2011).

35. Chen, H. et al. Arabidopsis CULLIN4-damaged DNA binding protein 1 interacts with CONSTITUTIVELY PHOTOMORPHOGENIC1-SUPPRESSOR OF PHYA complexes to regulate photomorphogenesis and flowering time. Plant Cell 22, 108-123 (2010).

36. Bernhardt, A. et al. CUL4 associates with DDB1 and DET1 and its downregulation affects diverse aspects of development in Arabidopsis thaliana. Plant J. 47, 591-603 (2006)

37. Osterlund, M. T., Hardtke, C. S., Wei, N. \& Deng, X. W. Targeted destabilization of HY5 during light-regulated development of Arabidopsis. Nature 405, 462-466 (2000).

38. Bauer, D. et al. Constitutive photomorphogenesis 1 and multiple photoreceptors control degradation of phytochrome interacting factor 3, a transcription factor required for light signaling in Arabidopsis. Plant Cell 16, 1433-1445 (2004)

39. Benvenuto, G., Formiggini, F., Laflamme, P., Malakhov, M. \& Bowler, C. The photomorphogenesis regulator DET1 binds the amino-terminal tail of histone H2B in a nucleosome context. Curr. Biol. 12, 1529-1534 (2002).

40. Brickner, J. H. \& Walter, P. Gene recruitment of the activated INO1 locus to the nuclear membrane. PLoS Biol. 2, e342 (2004).

41. Sarma, N. J. et al. Glucose-responsive regulators of gene expression in Saccharomyces cerevisiae function at the nuclear periphery via a reverse recruitment mechanism. Genetics 175, 1127-1135 (2007).

42. Casolari, J. M. et al. Genome-wide localization of the nuclear transport machinery couples transcriptional status and nuclear organization. Cell 117, 427-439 (2004)

43. Dieppois, G., Iglesias, N. \& Stutz, F. Cotranscriptional recruitment to the mRNA export receptor Mex67p contributes to nuclear pore anchoring of activated genes. Mol. Cell Biol. 26, 7858-7870 (2006).

44. Brickner, D. G. et al. Transcription factor binding to a DNA zip code controls interchromosomal clustering at the nuclear periphery. Dev. Cell 22, 1234-1246 (2012).

45. Ahmed, S. et al. DNA zip codes control an ancient mechanism for gene targeting to the nuclear periphery. Nat. Cell Biol. 12, 111-118 (2010).

46. Light, W. H., Brickner, D. G., Brand, V. R. \& Brickner, J. H. Interaction of a DNA zip code with the nuclear pore complex promotes H2A.Z incorporation and INO1 transcriptional memory. Mol. Cell 40, 112-125 (2010).
47. Liu, X. et al. PHYTOCHROME INTERACTING FACTOR3 associates with the histone deacetylase HDA15 in repression of chlorophyll biosynthesis and photosynthesis in etiolated Arabidopsis seedlings. Plant Cell 25, 1258-1273 (2013).

48. Tessadori, F. et al. Phytochrome B and histone deacetylase 6 control lightinduced chromatin compaction in Arabidopsis thaliana. PLoS Genet. 5, e1000638 (2009)

49. Lau, O. S. et al. Interaction of Arabidopsis DET1 with CCA1 and LHY in mediating transcriptional repression in the plant circadian clock. Mol. Cell 43, 703-712 (2011).

50. Nagatani, A., Reed, J. W. \& Chory, J. Isolation and initial characterization of Arabidopsis mutants that are deficient in phytochrome A. Plant Physiol. 102, 269-277 (1993).

51. Reed, J. W., Nagpal, P., Poole, D. S., Furuya, M. \& Chory, J. Mutations in the gene for the red/far-red light receptor phytochrome B alter cell elongation and physiological responses throughout Arabidopsis development. Plant Cell 5, 147-157 (1993).

52. Lian, H. L. et al. Blue-light-dependent interaction of cryptochrome 1 with SPA1 defines a dynamic signaling mechanism. Genes Dev. 25, 1023-1028 (2011).

53. Leivar, P. et al. Multiple phytochrome-interacting bHLH transcription factors repress premature seedling photomorphogenesis in darkness. Curr. Biol. 18, 1815-1823 (2008).

54. Su, Y. S. \& Lagarias, J. C. Light-independent phytochrome signaling mediated by dominant GAF domain tyrosine mutants of Arabidopsis phytochromes in transgenic plants. Plant Cell 19, 2124-2139 (2007).

55. Chen, M. et al. Arabidopsis HEMERA/pTAC12 initiates photomorphogenesis by phytochromes. Cell 141, 1230-1240 (2010).

56. Schubert, I., Dolezel, J., Houben, A., Scherthan, H. \& Wanner, G. Refined examination of plant metaphase chromosome structure at different levels made feasible by new isolation methods. Chromosoma 102, 96-101 (1993).

57. Xu, X. M. et al. NUCLEAR PORE ANCHOR, the Arabidopsis homolog of Tpr/ $\mathrm{Mlp} 1 / \mathrm{Mlp} 2 /$ megator, is involved in mRNA export and SUMO homeostasis and affects diverse aspects of plant development. Plant Cell 19, 1537-1548 (2007)

58. Jasencakova, Z., Meister, A., Walter, J., Turner, B. M. \& Schubert, I. Histone H4 acetylation of euchromatin and heterochromatin is cell cycle dependent and correlated with replication rather than with transcription. Plant Cell 12, 2087-2100 (2000).

59. Jasencakova, Z., Meister, A. \& Schubert, I. Chromatin organization and its relation to replication and histone acetylation during the cell cycle in barley. Chromosoma 110, 83-92 (2001).

60. R Development Core Team. $R$ : A language and environment for statistical computing. Retrieved from http://www.R-project.org (R Foundation for Statistical Computing, Vienna, Austria, 2013).

61. Galvão, R. M. et al. Photoactivated phytochrome interact with HEMERA and promote its accumulation to establish photomorphogenesis in Arabidopsis. Genes Dev. 26, 1851-1863 (2012).

\section{Acknowledgements}

We thank Drs Sam Johnson and Yasheng Gao from the Duke Light Microscopy Core Facility for their help with fluorescence imaging and data analysis. We are grateful to P. Andrew Nevarez for critical reading of the manuscript. We thank Dr Mats Nilsson for his help with the padlock FISH protocol and Dr Sara Henriksson for introducing padlock FISH to M.C. We thank Dr Peter Quail for the pifq mutant and the anti-PHYB antibody, Dr Joanne Chory for the det1-1 mutant, Dr Xing-Wang Deng for the cop1-4 mutant, Dr Hongqian Yang for the hy5 mutant and Dr Steven Henikoff for the anti-HTR12 antibody. M.C. is grateful for all of the instructors of the 2007 FISH course at the Cold Spring Harbor Laboratory, including Drs Viki Allan, Ke Hu, Sui Huang, John Murray and Jennifer Waters. This research was supported by grants from the National Institutes of Health R01 GM087388 and the National Science Foundation IOS-1051602 to M.C.

\section{Author contributions}

C.-M.F. and M.C. designed the experiments. C.-M.F. performed the majority of the experiments and data analysis. Y.Q., E.K.V. and E.J.Y. contributed to the experiments. C.M.F. and M.C. wrote the manuscript.

\section{Additional information}

Supplementary Information accompanies this paper at http://www.nature.com/ naturecommunications

Competing financial interests: The authors declare no competing financial interests.

Reprints and permission information is available online at http://npg.nature.com/ reprintsandpermissions/

How to cite this article: Feng, C.-M. et al. Light-regulated gene repositioning in Arabidopsis. Nat. Commun. 5:3027 doi: 10.1038/ncomms4027 (2014). 